\title{
Trie Size in a Dynamic List Structure
}

\author{
G. Louchard \\ Université Libre de Bruxelles, Département d'Informatique-CP 212 \\ Boulevard du Triomphe - 1050 Brussels, Belgium
}

\begin{abstract}
This paper considers a classical binary tree implementation of a set of keys: the trie. The trie size properties in a static environment are well known: the size is asymptotically Gaussian when the keys number gets large. In this paper we analyze the trie in a dynamic environment, where the trie is allcwed to grow and shrink in a probabilistic way. It appears that the trie size can be described by a stocliastic process which is asymptotically non-Markovian Gaussian. This also allows the complete asymptolic analysis of the trie size maximum and the trie size integrated cost.
\end{abstract}

\section{Introduction}

List structures are well known dynamic objects in Computer Science. Let us mention dictionaries, priority queues, symbol tables, linear lists, stacks, tries, etc.

Many of them have already been analysed in the literature: see for instance Flajolet et al. [7], [6], Louchard [11], Louchard et al. [13]. Here we analyze the dymamic trie (which is a classical binary tree implementation of a set of keys), i.e. a trie initially of size 0 and returning to size 0 at step $2 n$, on which insertions and deletions are performed. We want to characterize the trie size, i.e. the trie internal node number $N$ as a stochastic process. $N$ has already been stalically investigaled (i.e. with a fixed lotal keys number $n$ ) in Regnier, Jacquel [8] : it appears that $N$ is asymptotically equivalent $(n-\infty)$ to a Gaussian random variable. For the dynamic trie size, we obtain here a Gaussian non-Markovian process. This also allows the analysis of two crucial variables in random structure complexity: the trie size maximum and the trie size cost (total integrated size on $[0,2 n]$ ).

Apart from the Knuth model (see [14]), this is the first time we meet a dynamic list structure with a non-Markovian behaviour.

The paper is organized as follows: Section 2 recalls trie definition and keys structure properlies. Section 3 and 4 analyze the effects of an insertion and a deletion. Section 5 is devoted to a sequence of $n$ insertions: several variables of interest are investigated. Section 6 deals with the covariance in the case of consecutive insertions. Section 7 gives a complete characterization of the trie size process. Section 8 deals wilh trie size maximum and section 9 with the trie size cost function. Section 10 concludes the paper.

The tools we use here are quite different from those we used in [12] (and of course different from classical combinatorial analysis). There, we were dealing with 
Laplace's asymptotic method on functional space. Here we view the trie as a partition of $[0,1]$ into intervals with exactly 1 or 0 key in each interval and we study the various (conditional) probabilities of $m$ keys falling into such intervals. Each key has a uniform $[0,1]$ distribution. This can be seen as an urn model, with dynamically varying urn sizes, where we study the occupancy distributions (For the classical occupancy problem, see Johnson and Kotz [10]). We mainly use difference equations on characteristic functions and detailed analysis of (conditioned) urn behaviour.

All detailed proofs and computations can be found in Louchard [15].

\section{Binary trie and histories}

Let us consider a classical binary trie where bits in the keys are 0 with probability $y$ (and 1 with probability $q$ ): the set of keys is represented by a binary tree where edges are labelled by 0 or 1 and leaves contain the keys. The access path from the root to a leaf is a minimal prefix of the corresponding key.

In this paper, we consider the symmetric case: $p=q=1 / 2$. We will use the trie to implement a dynamic list structure starling from $0 \mathrm{key}$ al time 0 and returning to 0 al step (or time) $2 n$.

lollowing Louchard [11] notations, let us first study the pos function for a classical keys structure with insertions (I) (each key has a uniform $[0,1]$ density) and deletions (D) (eaclı deleted key is choosen among $n$ existing keys with equal probability $1 / n$ ). The possibility function (defined for each request) is given by ( $n$ is the number of keys).

$$
\operatorname{pos}(10)
$$

\begin{tabular}{|c|c|c|c|}
\hline $\mathrm{I}$ & $\mathrm{D}$ & $\mathrm{Q}^{+}$ & $\mathrm{Q}^{-}$ \\
\hline $\mathrm{I}$ & $\mathrm{n}$ & 0 & 0 \\
\hline
\end{tabular}

Indeed no order is requested here: the trie only depends on key values. Moreover the insertion and deletion policy insures that the trie can be seen, at each time $t$ (with $n$ keys) as being constructed on $n$ independent keys. The process describing the number of keys $n$ at time $t$ is thus asymptotically equivalent to a priority queue $P . Q$. so that Theor. 5.3 from [11] gives the associated Gaussian process.

\section{Insertions}

In this section, we analyze the effect of an insertion $I$ on the configuration of the trie. The asymptotic distribution of a successful search in a trie is well known: see [12], [16] and [8].

Let us analyse the random variable (R.V.) $i$ : increase in the intemal nodes number $N$ ( $N$ is the trie size).

Assume first that we have no information about the trie, apart from its keys tolal number $n$. We shall use the same approach as in [12] Sec.6. The trie can be seen 
as a partition of $[0,1]$ into intervals (of size $1 / 2^{j}$ ) with exactly $\rfloor$ or $O$ key in each interval.

Each interval $1 / 2^{j}$ possess one "buddy" of the same size. For instance a leaf $*$ of the trie is represented as follows (the interval is pictured as a rectangular box).

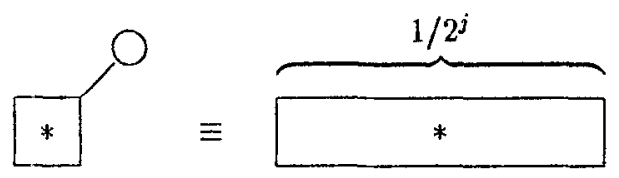

i) Assume that the inserted key - falls into such an interval. It is clear that the new trie depends on which buddy (after successive bisections) will contain the new key •. By symmetry, only one case must be considered (the new key is pictured with $a \bullet$ ).

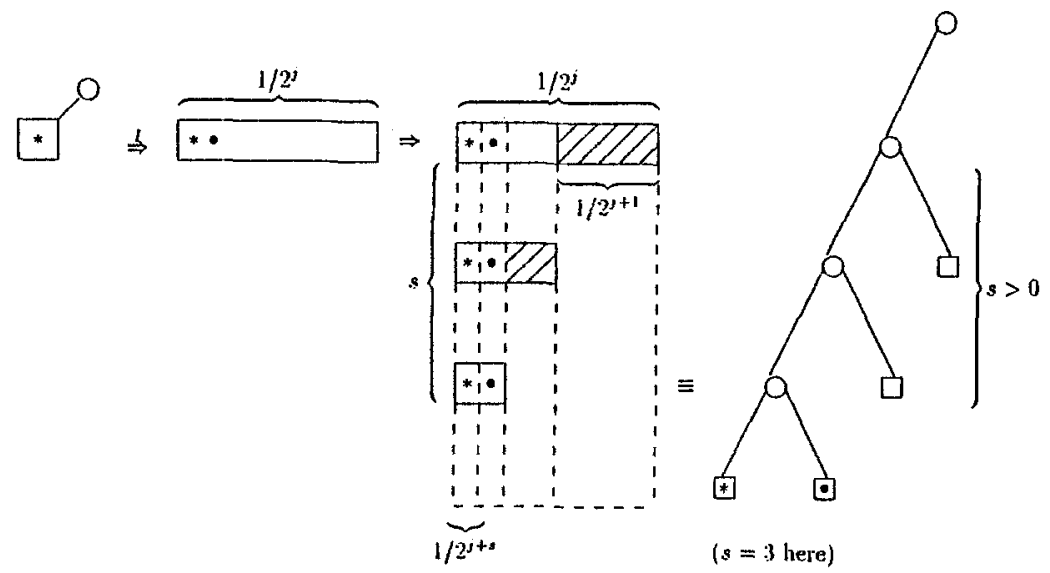

We obtain $\operatorname{Pr}[i=s]=\left(\frac{1}{2}\right)^{s}, s>0$. Note that we have created $(s-1)$ new empty leaves.

Apart from the inserted key $\bullet$, there are $n$ keys in the trie. The probability that none of these keys falls into an interval of size $1 / 2^{j}$ is given by a binomial. In first approximation this binomial is asymptotically Poisson, with probability $e^{-n / 2^{x}}$. However we will not use the Poissonization argument as in [12] and [17]: we should then have to de-Poissonize our results. We shall instead correct the Poisson approximation when necessary.

Case i) corresponds to ( $\diamond$ means that the exact status of this node is unimportant)

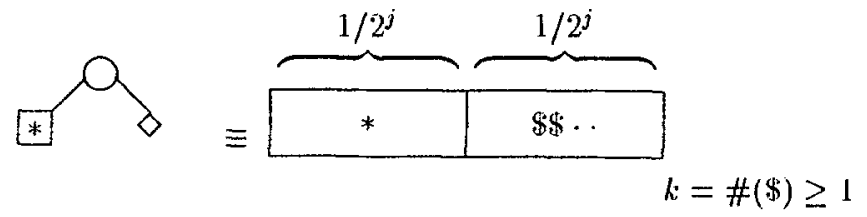


ii) the other subcase is related to

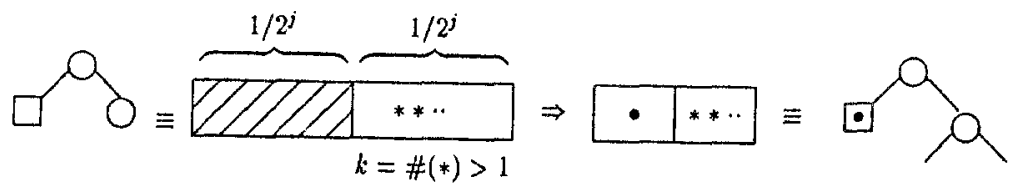

In case ii), increase $i \equiv 0$.

So we obtain for the two above-mentioned cases

i)

$$
\operatorname{Pr}[i=s] \sim\left\{\sum_{j=1}^{\infty} e^{-n / 2^{j}} \frac{n}{2 j}\left[1-e^{-n / 2^{j}}\right]\right\}\left(\frac{1}{2}\right)^{s}, s>0
$$

ii)

$$
\operatorname{Pr}[i=0] \sim\left\{\sum_{j=1}^{\infty} e^{-n / 2^{j}}\left[1-e^{-n / 2^{j}}\left[1+\frac{m}{2^{j}}\right]\right]\right\}
$$

From these formula's we can deduce unconditional mean $\bar{i}$ and variance $\sigma_{i}^{2}$.

Let us now define the real problem. At lime $t$, we know only the keys total number $n$ and the trie size $N$. But the insertion process is Markovian if we know also all key values (or at least the exacl partition of $[0,1]$ ). We can't deduce them from $n$ and $N$. Moreover, after increasing by one key, the new trie must have the same unconditional probabilistic properties as a trie build on $(n+1)$ independent keys. It is proved in [8] and [9] that the trie size $N$ after only $n$ insertions is asymptotically Gaussian. The explicit values of mean and variance of $N$ are given in [17].

\section{Deletions}

This section deals with the effect of a deletion $D$ on the trie's configuration. The R.V. $d$ (decrease in the internal nodes number $N$ ) is investigated. Assume first that we have no information about the trie apart from its key number $n$.

i) If the deleted key $\bullet$ is such that its leaf is like:

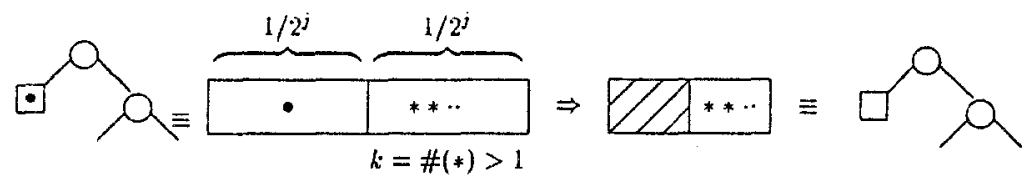

then we observe no effect on the trie size $N, d=0$. 
ii) If the deleted key $\bullet$ is such that ( $\diamond$ means that the exact status of this node is unimportanli):

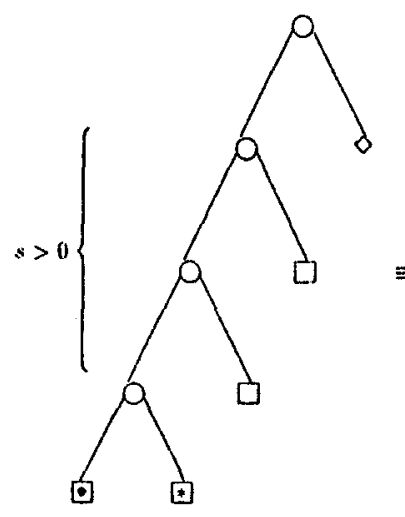

$\Delta=1 / 2^{j}$

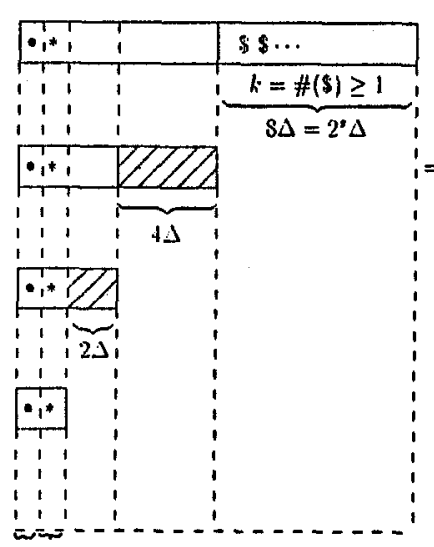

$\Delta \Delta$

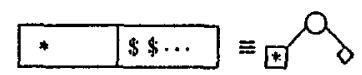

$(:=3$ here $)$
$\#(\square)=s-1$

We see that $d=-s$. Apart from the deleted key $\bullet$, there are $n-1$ other keys in the trie. Again, the probability that none of these keys falls into an interval of size $1 / 2^{j}$ is asymptotically given by $e^{-(n-1) / 2^{j}}$.

iii) So we obtain for the above-mentioned cases

$$
\begin{aligned}
\operatorname{Pr}[d=0] \sim & \left\{\sum_{j=1}^{\infty} e^{-(n-1) / 2^{j}}\left[1-e^{-(n-1) / 2^{j}}\left[1+\frac{(n-1)}{2^{j}}\right]\right]\right\} \\
\operatorname{Pr}[d=-s] \sim & \left\{\sum_{j=k+1}^{\infty} e^{-(n-1) / 2^{j}}\left[e^{-(n-1) / 2^{j}} \frac{(n-1)}{2^{j}}\right] e^{-\frac{(n-1)}{2 j} 2\left[2^{s-1}-1\right]}\right. \\
& {\left.\left[1-e^{\frac{-(n-1)}{2 j} \cdot 2^{s}}\right]\right\} }
\end{aligned}
$$

with $s>0$. From these formula's, we can deduce $\bar{d}$ and $\sigma_{d}^{2}$ (unconditioned values).

Again we meet the Markovian problem. If we only know $n$ and $N$, we can't deduce the exact partition of $[0,1]$.

\section{A sequence of $n$ insertions}

In this section, we study the effect of a sequence of $n$ insertions on several variables of interest: the total number $S_{n}^{e}$ of empty leaves, $D_{n}^{e}$; the empty part of $[0,1]$ (i.e. the total size of empty leaves), $D_{n}^{\prime}$ (full leaves). We rediscover (by another method) 
the asymptotic properties of $S_{n}^{e}$ (already analysed in [17]). This analysis also paves the way to all subsequent covariance computations.

Let us first set a few notations:

- \#f $(l):=$ number of full leaves (i.e. with onc key) of size $1 / 2^{\ell}$

- $\#^{e}(\ell):=$ number of cmply leaves (i.e. with $0 \mathrm{key}$ ) of size $1 / 2^{\ell}$

- $c^{\bullet}(l):=E\left[H^{\bullet}(\ell)\right]$

- $D^{\bullet}=\sum_{j} \# \bullet(j) \frac{1}{2 i}$

i.e. $D^{e}:=$ empty parti of $[0,1]$

$D^{l}:=$ full part of $[0,1]$

Off course $D^{e}+D^{\prime}=1$.

- $S^{\bullet}=\sum_{j} \#^{\bullet}(j)$. For any quantity $Q, Q_{n}:=Q$ after $n$ insertions.

Obviously $S_{n}^{f} \equiv n$ and the total internal node number $N_{n}$ is given by:

$$
N_{n}=S_{n}^{e}+S_{n}^{f}-1=n+S_{n}^{e}-1
$$

Some expectations can now be considered. By (1) we know $\epsilon_{n}^{\bullet}$. Indeed, we deduce

$$
\left\{\begin{array}{l}
\epsilon_{n}^{f}(j) \frac{1}{2^{j}}=E\left[\#_{n}^{f}(j) \frac{1}{2^{j}}\right] \sim e^{-n / 2^{j}} \frac{n}{2^{j}}\left[1-e^{-n / 2^{j}}\right] \\
\epsilon_{n}^{c}(j) \frac{1}{2^{j}}=E\left[\#_{n}^{e}(j) \frac{1}{2^{j}}\right] \sim e^{-n / 2^{j}}\left[1-e^{-n / 2^{j}}\left[1+\frac{n}{2^{j}}\right]\right]
\end{array}\right.
$$

So that, for instance

$$
\left\{\begin{array}{l}
E\left(D_{n}^{e}\right) \sim \sum_{j=1}^{\infty}\left\{e^{-n / 2^{j}}\left[1-e^{-n / 2^{j}}\left[1+\frac{n b}{2^{j}}\right]\right]\right\} \\
E\left(S_{n}^{\epsilon}\right) \sim \sum_{j=1}^{\infty} 2^{j}\left\{e^{-n / 2^{j}}\left[1-e^{-n / 2^{j}}\left[1+\frac{n}{2^{j}}\right]\right]\right\}
\end{array}\right.
$$

$A$ few difference equations can now be established. Let $\Delta_{n} \epsilon_{n}^{\bullet}(\ell):=\epsilon_{n+1}^{\bullet}(\ell)-\epsilon_{n}^{\bullet}(\ell)$. Then we derive

$$
\left\{\begin{array}{l}
\Delta_{n} \epsilon_{n}^{j}(\ell)=\sum_{j=1}^{\ell-1}\left(\epsilon_{n}^{j}(j) \frac{1}{2^{j}}\right) \frac{1}{2^{\ell-j}} \cdot(2)+\left(\epsilon_{n}^{l}(\ell) \frac{1}{2^{\ell}}\right) \cdot(-1)+\epsilon_{n}^{e}(\ell) \frac{1}{2^{\ell}} \cdot(1) \\
\Delta_{n} \epsilon_{n}^{e}(\ell)=\sum_{j=1}^{\ell-1}\left(\epsilon_{n}^{l}(j) \frac{1}{2^{j}}\right) \sum_{k=\ell-j+1}^{\infty} \frac{1}{2^{k}} \cdot(1)+\left(\epsilon_{n}^{e}(\ell) \frac{1}{2^{\ell}}\right) \cdot(-1)
\end{array}\right.
$$

From (4), we derive

$$
\left\{\begin{array}{c}
\Delta_{n}\left[E\left(S_{n}^{f}\right)\right]=E\left(D_{n}^{f}\right)+E\left(D_{n}^{e}\right)=1 \quad \text { (as it should) } \\
\Delta_{n}\left[E\left(S_{n}^{e}\right)\right]=E\left(D_{n}^{f}\right)-E\left(D_{n}^{e}\right)=1-2 E\left(D_{n}^{e}\right) \\
\text { (this can be checked against (3)) }
\end{array}\right.
$$


To obtain the distribution of $S^{e}$ and $D^{e}$, let us analyze $\varphi_{n}\left(w_{1}\right):=E_{n}\left[e^{i w_{1} S_{n}^{e}}\right]$. We obtain the difference equation

$$
\begin{aligned}
\Delta_{n} \varphi_{n}\left(w_{1}\right) & =E\left\{D_{n}^{f}\left(\sum_{s=1}^{\infty} \frac{1}{2^{s}} e^{i w_{1}(s-1)}\right) e^{i w_{1} S_{n}^{e}}+D_{n}^{e} e^{-i w_{1}} e^{i w_{1} S_{n}^{e}}-e^{i w_{1} S_{n}^{e}}\right\} \\
& =E\left\{D_{n}^{J} \frac{1}{2} \frac{e^{i w_{1} S_{n}^{e}}}{1-e^{i w_{1} / 2}}+D_{n}^{e} e^{-i w_{1}} e^{i w_{1} S_{n}^{e}}-e^{i w_{1} S_{n}^{e}}\right\}
\end{aligned}
$$

Expanding, we derive

$$
\begin{aligned}
i w_{1}: \Delta_{n}\left[E\left(S_{n}^{e}\right]=\right. & E\left(D_{n}^{f}\right)-E\left(D_{n}^{e}\right) \quad(\text { which confirms (5)) } \\
\frac{-w_{1}^{2}}{2}: \Delta_{n}\left[E\left[\left(S_{n}^{e}\right)^{2}\right]\right]= & 2 E\left(D_{n}^{f} S_{n}^{e}\right)+3 E\left(D_{n}^{f}\right) \\
& -2 E\left(D_{n}^{e} S_{n}^{e}\right)+E\left(D_{n}^{e}\right)
\end{aligned}
$$

We now turn to the asymptotic analysis of $E\left(D_{n}^{e}\right), E\left(S_{n}^{e}\right)$ and $\operatorname{VAR}\left(S_{n}^{e}\right)$. We obtain the following result

\section{Theorem 5.1}

$$
\begin{aligned}
E\left(D_{n}^{\epsilon}\right) & \sim 1-\frac{1}{2 \ln 2}+\beta_{0}(n) \\
E\left(D_{n}^{f}\right) & \sim \frac{1}{2 \ln 2}-\beta_{0}(n) \\
E\left(S_{n}^{\epsilon}\right) & \sim n\left[\alpha_{0}+\beta_{1}(n)\right]
\end{aligned}
$$

with $\alpha_{0}=\frac{1}{\ln _{2}}-1$ and $\beta_{0}(n)$ are (small) periodic function of $\log _{2} n$

$$
V A R\left(S_{n}^{e}\right) \sim n a_{2}
$$

with $\alpha_{2}:=1-\frac{3}{2 \ln 2}-\alpha_{0}^{2}+2 \sum_{c=1}^{\infty} \frac{\delta}{1+\delta} / \ln 2$, and $\delta:=1 / 2^{\ell}$.

Proof The detailed prool, based on somewhere delicate urn sizes analysis, is given in $[15]$.

We now tum to the asymptotic distribution of $S_{11}^{e}$. We obtain the following theorem (the Gaussian character of $S_{n}^{e}$ is proved in [8] and [9] by a different method).

Theorem $5.2 S_{n}^{e}$ is asymptotically Gaussian with mean $\sim \alpha_{0} n$ and $V A R \sim n \alpha_{6}$.

Our proof is again based on some difference equation properties (the details are given in [15]).

\section{Covariances - The case of consecutive insertions}

This section is devoted to the case of $\nu$ consecutive insertions: we analyze the covariance between $S_{n_{1}}^{e}$ et $S_{n_{2}}^{e}$. This covariance is given by the following theorem. 
Theorem 6.1 Let $\operatorname{COV}\left(S_{n_{1}}^{e}, S_{n_{2}}^{e}\right) \sim \Psi\left(t_{1}, t_{2}\right) n$, with $n_{1}=n t_{1}, n_{2}=n t_{2}, n_{2}=$ $u_{1}+\nu,(\nu \geq 0)$.

Then $\Psi\left(t_{1}, t_{2}\right)=\Pi\left(\frac{t_{2}}{t_{1}}\right) \cdot t_{2}$ where

$$
\begin{aligned}
\mathrm{Il}(z) & =-\sum_{\ell=1}^{\infty} \delta\left[\delta^{3} z-3 \delta^{3} z^{2}+2 \delta^{3} z^{3}-4 \delta^{2} z+\delta^{2}+2 \delta^{2} z^{4}+3 \delta^{2} z^{2}-2 \delta^{2} z^{3}\right. \\
& \left.-3 \delta z^{2}-\delta z+4 z^{3} \delta-1+z^{2}\right] /[(1+\delta z)(1+\delta)(z+\delta)(1+\delta z-\delta) z \ln (2)] \\
& -\frac{1}{2} \frac{\left(\ln (2) z-2 \ln (2)+\ln (2) z^{2}+2-2 z^{2}\right)}{\ln (2)^{2}(z+1) z}+\alpha_{2}
\end{aligned}
$$

$\left(\alpha_{2}\right.$ as given in Theor. 5.1, $\left.\delta:=1 / 2^{l}\right)$.

Proof The detailed proof is again given in [15].

\section{Trie size in a dynamic structure}

This section contains the main result of this paper: a complete characterization of the trie size process. We first recall the keys structure characteristics. Then we analyse a static structure, with $I$ as first operation. $D$ is described next. The static structure covariance is computed and finally the full dynamic structure covariance is obtained.

\subsection{Key total number process}

From Louchard [11] Theorem 5.3, it is known that the priority queue $Y$ describing the number of keys at time $n t$ is such that

$$
\frac{Y([n t])-n f_{1}(t)}{\sqrt{n}} \Rightarrow X(t), \quad 0 \leq t \leq 2
$$

where we assume that we start with an empty structure at time 0 and we return to an empty structure at time $2 n$. We have obtained in [11]:

$$
f_{1}(t)=\frac{1}{2} t(2-t)
$$

$X(t)$ is a. Markovian Gaussian process with covariance

$$
\begin{aligned}
f_{2}(s, t) & =\gamma(s) \gamma(2-t), & & s \leq t \\
& =\gamma(t) \gamma(2-s), & & s \geq t
\end{aligned}
$$

and $\gamma(v)=v^{2} / 2$.

The error term in (7) can be deduced from the various expansions in [11]: it appears that the relative error in the density is $O\left(\frac{1}{\sqrt{n}}\right)$. In the sequel, we will always 
use the denotation structure for the process (7). Keys total number is usually denoted by $n_{1}, n_{2}, n_{t}$. If we denote by $n_{1}, n_{2}$ the number of keys at time $t_{1}, l_{2}$, we have

$$
\begin{aligned}
& E\left[e^{i\left(\zeta_{1} n_{1}+\zeta_{2} n_{2}\right)}\right] \sim \\
& \quad \exp \left[n \left\{i\left[\zeta_{1} f_{1}\left(\iota_{1}\right)+\zeta_{2} f_{1}\left(\iota_{2}\right)\right]\right.\right. \\
& \left.\left.\quad-\frac{1}{2}\left[\zeta_{1}^{2} f_{2}\left(\iota_{1}, t_{1}\right)+2 \zeta_{1} \zeta_{2} f_{2}\left(t_{1}, \iota_{2}\right)+\zeta_{2}^{2} f_{2}\left(t_{2}, t_{2}\right)\right]\right\}\right]
\end{aligned}
$$

For further use, we denote

$$
\begin{aligned}
& n_{1}^{*}:=n f_{1}\left(l_{1}\right) \\
& n_{2}^{*}:=n f_{1}\left(t_{2}\right)
\end{aligned}
$$

\subsection{Non-random static structure}

Let us start with a non-random (NR) stalic structure, following a path fixed by $n f_{1}(l)$. Let us divide the $2 n$ steps into $2 n / m$ groups of $m$ steps, when $m$ is large and $m=o(n)$. For instance, choose $D t$ small such that $m=n(D t)$. It is well known (see Louchard [11], Lemma 5.1) that the probability $p_{I}$ of an inser'ion is asymptotically constant on such an interval and is given by

$$
p_{I} \sim \frac{1}{2}\left(1+f_{1}^{\prime}(t)\right)=1-t / 2
$$

similarly

$$
p_{D} \sim \frac{1}{2}\left(1-f_{1}^{\prime}(t)\right)=t / 2
$$

The total number of insertions (deletions) on $D l$ is given by $n_{I}=m p_{I}\left(n_{D}=m p_{D}\right)$. Choose an interval $\Delta\left(=1 / 2^{j}\right)$ in $[0,1]$ such that $n_{t}^{*} \Delta=O(1) \cdot\left(n_{t}^{*}=n f_{1}(t)\right)$. As the number of inserted keys in $\Delta$ (or deleted keys) is very small compared with $n_{t}^{*} \Delta$, we see that, asymptotically, insertions and deletions perform independently on $\Delta$. Assume that, among the $n_{l}^{*}$ keys, $n_{\Delta}$ stay in $\Delta$. The probability that $\nu_{\Delta}^{D}$ of these keys are removed is given by

$$
\frac{\left(\begin{array}{c}
n_{\Delta} \\
\nu_{\Delta}^{D}
\end{array}\right)\left(\begin{array}{c}
n_{l}^{*}-n_{\Delta} \\
n_{D}-\nu_{\Delta}^{D}
\end{array}\right)}{\left(\begin{array}{c}
n_{\imath}^{*} \\
n_{D}
\end{array}\right)}
$$

By Stirling's formula, this probability is seen to be, asymptotically

$$
\left(\begin{array}{l}
n_{\Delta} \\
\nu_{\Delta}^{D}
\end{array}\right) p^{\nu_{\Delta}^{D}}(1-p)^{n_{\Delta}-\nu_{\Delta}^{D}}
$$

with $p=\frac{n_{D}}{n_{i}^{*}}=\frac{p_{D}(D t)}{f_{1}(t)}$.

This is a Binomial wilh very small $p$ (Bin law).

Also $\nu_{\Delta}^{I}$ : the number of keys among the $n_{I}$ inserted keys, which fall inside $\Delta$ is asymplotically given by a Poisson law ( $\mathcal{P}$ law)

$$
e^{-n_{I} \Delta} \frac{\left(n_{I} \Delta\right)^{\nu_{\Delta}^{I}}}{\left(\nu_{\Delta}^{I}\right) !}
$$


with very small parameter $n_{I} \Delta=p_{I} n \Delta(D t)$. We are thus led to a classical birth and death process, with birth rate $\lambda(l):=n \Delta(1-l / 2)$ and death rate $\mu(l):=\frac{1}{2-t}$. The survival probability between times $t_{1}$ and $t_{2}$ is given by $p s_{2,1}=e^{-\int_{t_{1}}^{t_{2}} \mu(s) d s}=\frac{2-t_{2}}{2-t_{1}}$ and $\lim _{t_{3} \rightarrow t_{1}} p s_{2,1}=1$. The tolal number of inserted keys (into $\Delta$ ), between $t_{1}$ and $t_{2}$, which have not been deleted at $t_{2}$ is Poisson with parameter $n_{2,1}$ given by

$$
n_{2,1}=\int_{t_{1}}^{t_{2}} \lambda(u) e^{-\int_{u}^{t_{2}} \mu(s) d s} d u=n \Delta\left(1-\frac{t_{2}}{2}\right)\left(t_{2}-t_{1}\right)
$$

which can also be written as

$$
n_{2,1}=\tilde{\gamma}_{2,1} y
$$

and

$$
\begin{aligned}
y & =n_{1}^{*} \Delta \\
\tilde{\gamma}_{2,1} & =\frac{\left(2-t_{2}\right)\left(t_{2}-t_{1}\right)}{t_{1}\left(2-t_{1}\right)}
\end{aligned}
$$

Or course $\lim _{t_{2} \rightarrow t_{1}} \dot{\gamma}_{2,1}=0$.

If, at time $\ell_{1}$, we have a fixed number $n_{\Delta}$ of keys inside $\Delta$, the total number of keys a.t $t_{2}$ in $\Delta$ is given by

$$
\operatorname{Bin}\left[n_{\Delta}, p s_{2,1}\right]+\mathcal{P}\left[n_{2,1}\right]
$$

If, at time $t_{1}$, we have a random $\mathcal{P}\left[n_{1}^{*} \Delta\right]$ number of keys inside $\Delta$, it is easy to check that we are led to $P\left[n_{2}^{*} \Delta\right]$ keys in $\Delta$ at time $t_{2}$. This is also obvious from probabilistic arguments. For further use, set

$$
\frac{n_{2}^{*}}{n_{1}^{*}}=1+\gamma_{2,1}=\frac{t_{2}\left(1-t_{2} / 2\right)}{t_{1}\left(1-t_{1} / 2\right)}
$$

To ease notations, we shall write $\tilde{\gamma}, \gamma$ and $p s$ (without indices).

We are now ready to analyse the covariance $C O V_{N R}\left(S_{i_{1}}^{e}, S_{l_{2}}^{e}\right)=\Psi_{N R}\left(t_{1}, t_{2}\right) n$ say. We must consider two subcases: starting with an insertion or with a deletion. Each subcase leads to a function $f_{3}^{l}\left(t_{1}, t_{2}\right)$ (or $f_{3}^{D}\left(t_{1}, t_{2}\right)$ ) that we have obtained in [15] as rather complicated integrals. The differential equation for $\Psi_{N R}\left(t_{1}, l_{2}\right)$ (Non-random structure) can be written as follows. As in Sec.6, we now obtain $\partial_{t_{1}} \Psi_{N R}\left(t_{1}, t_{2}\right)=f_{3, N R}\left(t_{1}, t_{2}\right)$ where

$$
f_{3, N R}\left(t_{1}, t_{2}\right)=p_{I} f_{3}^{I}\left(t_{1}, t_{2}\right)+p_{D} f_{3}^{D}\left(t_{1}, t_{2}\right)
$$

with $p_{I}=1-t_{1} / 2, p_{D}=l_{1} / 2$.

And we finally obtain the following theorem

Theorem 7.1 In a non-random struclure, the covariance $\operatorname{COV}\left(S_{t_{1}}^{e}, S_{i_{2}}^{e}\right) \sim \Psi_{N R}\left(t_{1}, t_{2}\right) n$ with

$$
\Psi_{N R}\left(t_{1}, t_{2}\right)=-\int_{t_{1}}^{t_{2}} f_{3, N R}\left(u, t_{2}\right) d u+\alpha_{2} f_{1}\left(t_{2}\right)
$$

and $f_{3}$ is given by $(9)$. 
Up to now, we have been unable (even with MAPLE) to get a close expression for $\Psi_{N R}\left(t_{1}, t_{2}\right)$. However, the behaviour of $\Psi_{N R}$ for $t_{2} \rightarrow t_{1}$ can be computed. Indeed, we can derive that

$$
\begin{aligned}
& \lim _{t_{3} \rightarrow t_{1}} f_{3}^{l}\left(t_{1}, t_{2}\right)=\frac{\alpha_{3}}{2}+E\left(D^{e}\right)+3 E\left(D^{f}\right)-\alpha_{0}^{2} \\
& \lim _{t_{2} \rightarrow t_{1}} f_{3}^{D}\left(t_{1}, t_{2}\right)=-\frac{\alpha_{3}}{2}
\end{aligned}
$$

with $a_{3}:=-5\left((2 \ln 2)+2 \sum_{\ell=1}^{\infty} \frac{\delta}{1+\delta} / \ln 2\right.$. Note that this can also be obtained from Sec.6.

Indeed, by simple probabilistic reasoning, we must have

$$
\begin{aligned}
& f_{3}^{I}\left(t_{1}, l_{1}\right)=\partial_{1} \Psi\left(t_{1}, l_{1}\right)=f_{3}(0) \\
& f_{3}^{D}\left(l_{1}, l_{1}\right)=-\partial_{t_{3}} \Psi\left(\iota_{1}, l_{1}\right)=-\left[a_{2}-f_{3}(0)\right]
\end{aligned}
$$

which lead to (10). This gives

$$
\begin{aligned}
\left.\partial_{t_{1}} \Psi_{N R}\left(t_{1}, t_{2}\right)\right|_{t_{1}=t_{2}} & =p_{I} f_{3}^{J}\left(t_{1}, t_{1}\right)+p_{D} f_{3}^{D}\left(t_{1}, t_{1}\right)=f_{1}^{\prime} \frac{\alpha_{3}}{2}+\frac{1}{2}\left(1+f_{1}^{\prime}\right)\left(m_{2}-\alpha_{0}^{2}\right) \\
\left.\dot{\partial}_{t_{2}} \Psi_{N R}\left(t_{1}, t_{2}\right)\right|_{t_{1}=t_{2}} & =p_{I}\left(-f_{3}^{D}\left(t_{1}, t_{1}\right)\right)+p_{D}\left(-f_{3}^{I}\left(t_{1}, t_{1}\right)\right) \\
& =f_{1}^{\prime} \frac{\alpha_{3}}{2}-\frac{1}{2}\left(1-f_{1}^{\prime}\right)\left(m_{2}-\alpha_{0}^{2}\right)
\end{aligned}
$$

This last result is of course compatible wilh Theor.7.1.

\subsection{The trie size in a dynamic structure}

Jet $N([n s])$ be the size of the brie at time s, By (2), we kllow that $N([n s])=$ $n+S_{[n s]}^{c}+1$.

We know that, al $t_{1}, l_{2}$, the size $n_{1}, n_{2}$ of the underlying structure is characterized by $(7)$, i.e. we can wrile

$$
n_{1} \sim n\left[f\left(t_{1}\right)+\frac{\theta_{1}}{\sqrt{n}}\right]+O(1), u_{2} \sim \eta\left[f\left(t_{2}\right)+\frac{\theta_{2}}{\sqrt{n}}\right]+O(1)
$$

where $\theta_{1}, \theta_{2}$ are Gaussian R.V., with mean 0 and covariance $f_{2}\left(t_{1}, t_{2}\right)$.

Given $n_{1}, n_{2}$, the size of the structure at $v \in\left(t_{1}, t_{2}\right)$ is given by $n z(v)$ where

$$
z(v)=f_{1}(v)+\varphi_{1}\left(\iota_{1}, t_{2}, v\right) \frac{\theta_{1}}{\sqrt{n}}+\varphi_{2}\left(l_{1}, t_{2}, v\right) \frac{\theta_{2}}{\sqrt{n}}+\frac{x(v)}{\sqrt{n}}+O\left(\frac{1}{n}\right)
$$

where $\varphi_{1}, \varphi_{2}$ can be computed as in Louchard [11] Sec.4.5.2 and $\chi(v)$ is a Gaussian process, with mean $0, \lambda\left(t_{1}\right)=\chi\left(t_{2}\right)=0$ and a covariance that can be deduced from $f_{2}$. After carefully checking the effect of these nodificalion on our computations, it appears that, conditionally on $n_{1}, n_{2}$, the covariance $\operatorname{COV}_{C}\left(S_{n_{1}}^{e}, S_{n_{2}}^{e}\right)$ is given by ${ }^{*} \Psi_{C}\left(t_{1}, l_{2}\right)$, will

$$
\Psi_{C}\left(t_{1}, t_{2}\right)=\Psi_{N R}\left(t_{1}, t_{2}\right)+\bar{\varphi}_{1}\left(t_{1}, t_{2}\right) \frac{\theta_{1}}{\sqrt{n}}+\bar{\varphi}_{2}\left(t_{1}, t_{2}\right) \frac{\theta_{2}}{\sqrt{n}}+O\left(\frac{1}{n}\right)
$$


for some $\bar{\varphi}_{1}, \bar{\varphi}_{2}$. lndeed, all functions used in the computation of $\Psi_{C}$ and involving $z^{\prime}(v)$ (instead of $f_{1}^{\prime}(v)$ ) can be rewritten in terms of $z(v), z\left(t_{1}\right)$ and $z\left(t_{2}\right)$; the properties of $\chi$ induce a 0 contribution (up to order $\frac{1}{\sqrt{n}}$ ). (we cannot use $\chi^{\prime}(t)$ as $\chi(\cdot)$ is a non-differentiable process, but integrating by parts provide correct expressions).

Now, given $n_{1}$ and $n_{2}$, Theorem 5.2 and (13) lead to

$$
\begin{aligned}
& E\left[e^{i\left(\kappa_{1} S_{n_{1}}^{e}+\kappa_{2} S_{n_{2}}^{c}\right)}\right] \\
& \quad \sim \exp \left\{i\left[\alpha_{0} n_{1} \kappa_{1}+\alpha_{0} n_{2} \kappa_{2}\right]-\frac{1}{2}\left[\alpha_{2} n_{1} \kappa_{1}^{2}+2 n \Psi_{C}\left(t_{1}, t_{2}\right) \kappa_{1} \kappa_{2}+\alpha_{2} n_{2} \kappa_{2}^{2}\right]\right\}
\end{aligned}
$$

The process $N([n s])$ is now characterized by the following theorem.

Theorem $7.2 N([n s])$ is a non-Markovian Ganssian process with $E[N([n s])] \sim$ $\alpha_{4} n f_{1}(s)$.

$$
\begin{aligned}
\Psi_{N}\left(s_{1}, s_{2}\right) & :=\operatorname{COV}\left[N\left(\left[n s_{1}\right]\right) N\left(\left[n s_{2}\right]\right)\right] \\
& \sim n\left[\alpha_{4}^{2} f_{2}\left(s_{1}, s_{2}\right)+\Psi_{N R}\left(s_{1}, s_{2}\right)\right] \\
\operatorname{VAR}[N([n s])] & \sim n\left[\alpha_{4}^{2} f_{2}(s, s)+a_{2} f_{1}(s)\right] \\
\alpha_{4} & :=a_{0}+1 .
\end{aligned}
$$

The relative error in lihe densily is $O\left(\frac{1}{\sqrt{n}}\right)$.

Proof From (7), (12) and (14), we obtain

$$
\begin{aligned}
& E\left[c^{i\left(\kappa_{1} N\left(\left[n t_{1}\right]\right)+\kappa_{2} N\left(\left[n t_{2}\right]\right)\right)}\right] \\
& \sim \exp \left\{i\left[\kappa_{1} \alpha_{4} n_{1}^{*}+\kappa_{2} \alpha_{4} n_{2}^{*}\right]-\frac{1}{2}\left[\alpha_{2} \kappa_{1}^{2} n_{1}^{*}+\alpha_{2} \kappa_{2}^{2} n_{2}^{*}+2 n \Psi_{N R}\left(t_{1}, t_{2}\right) \kappa_{1} \kappa_{2}\right]\right\} . \\
& \cdot E\left[\operatorname { e x p } \left\{0_{1} \sqrt{n}\left[i \alpha_{4} \kappa_{1}-\frac{1}{2} \alpha_{2} \kappa_{1}^{2}+\bar{\varphi}_{1}\left(t_{1}, l_{2}\right) \kappa_{1} \kappa_{2}\right]\right.\right. \\
& \left.\left.+\theta_{2} \sqrt{n}\left[i \alpha_{4} \kappa_{2}-\frac{1}{2} \alpha_{2} \kappa_{2}^{2}+\bar{\varphi}_{2}\left(t_{1}, t_{2}\right) \kappa_{1} \kappa_{2}\right]+O(1)\right\}\right]
\end{aligned}
$$

with $n_{1}^{*}:=n f_{1}\left(t_{1}\right), n_{2}^{*}:=f_{1}\left(t_{2}\right)$. The expectation leads, by $(8)$, to

$$
e^{\left.-n / 2\left[f_{2}\left(t_{1}, t_{1}\right) \zeta_{1}^{2}+2 f_{2}\left(t_{1}, t_{2}\right) \zeta_{1} \zeta_{2}\right]+f_{2}\left(t_{2}, l_{2}\right) \zeta_{2}^{2}\right]}
$$

where

$$
\begin{aligned}
& \zeta_{1}=\left[\alpha_{4} \kappa_{1}+\frac{i}{2} \alpha_{2} \kappa_{1}^{2}-i \bar{\varphi}_{1}\left(t_{1}, t_{2}\right) \kappa_{1} \kappa_{2}\right] \\
& \zeta_{2}=\left[\alpha_{4} \kappa_{2}+\frac{i}{2} \alpha_{2} \kappa_{2}^{2}-i \bar{\varphi}_{2}\left(t_{1}, t_{2}\right) \kappa_{1} \kappa_{2}\right]
\end{aligned}
$$

(15) becomes

$$
\begin{aligned}
\exp \{ & -11 / 2\left[f_{2}\left(l_{1}, l_{1}\right) \alpha_{4}^{2} \kappa_{1}^{2}+2 l_{2}\left(l_{1}, l_{2}\right) \alpha_{4}^{2} \kappa_{1} \kappa_{2}+I_{2}\left(l_{2}, l_{2}\right) \alpha_{4}^{2} \kappa_{2}^{2}\right. \\
& \left.\left.\left.+O \text { (cubic terms in } \kappa_{1}, \kappa_{2}\right)+O\left(\frac{1}{\sqrt{n}}\right)\right]\right\}
\end{aligned}
$$

Completion of the proof is now inmediate. 


\section{Trie size maximum}

To analyse the size maximum, we shall use a technique based on Daniels' results. Let a Gaussian process $Z(t)$ superimposed on a curve $\tilde{y}(t)$. If we look for $m:=$ $\max [Z(l)+\tilde{y}(t)]$ and the time $t^{*}$ at which this maximum occurs, it is equivalent to search for the hitting time of $Z(t)$ to the absorbing boundary $m-\tilde{y}(t)$. It is well known (see Durbin [4]) that, near the crossing point, $Z(t)$ behaves locally like a Brownian Motion B.M. (or a variant of it, such as a Brownian Bridge B.B.).It is also known that the hithing time and place densities for a B.B. can be deduced from the hitling time density for a B.M. (see for instance Louchard [14] for a constant boundary and Csaki et al [1] for a general proof).

Assume that $\tilde{y}(t)$ is given by

$$
\dot{y}(t)=\sqrt{n} y(t), \quad n \gg 1
$$

and that it has a unique maximum at $\bar{t}$, with $y(\bar{t})=0$. Daniels and Skyrme [2] have computed the asymptotic hitting time and place density.

Daniel's formula leads to the following theorem.

Theorem 8.1

$$
M:=\max _{[0,2]} N([n t]) \sim \frac{\alpha_{4} n}{2}+\sqrt{n} m+O\left(n^{1 / 6}\right)
$$

where $m$ and $t^{*}$ densities are delailed in [15] ( $m$ is asymplotically Gaussian).

\section{Trie size cost}

The integrated trie size cost is defined by

$$
\sigma^{*}:=\sum_{1}^{2 n} N(i) \sim n \int_{0}^{2} N([n s]) d s
$$

$\sigma^{*}$ is characterized by the following theorem

Theorem 9.1

$$
\frac{\sigma^{*}-n^{2} \nu_{1}}{\left(n^{3} \nu_{2}\right)^{1 / 2}} \sim \mathcal{N}(0,1)
$$

where $\mathcal{N}(0,1)$ is a Gaussian R.V.

$$
\begin{aligned}
& \nu_{1}:=\int_{0}^{2} \alpha_{4} f_{1}(s) d s=\alpha_{4} \frac{2}{3} \\
& \nu_{2}:=2\left[\int_{0}^{2} d s_{1} \int_{s_{1}}^{2} d s_{2} \Psi_{N R}\left(s_{1}, s_{2}\right)\right]+\alpha_{4}^{2} 8 / 45
\end{aligned}
$$

Proof The asymptolic mean is immediate from Theor.7.1. The R.V. $\int_{0}^{2} N([n s]) d s$ is a classical stochastic integral, which is Gaussian. Its variance is computed from theorem 7.1 and from [11] Theor.5.4 for the $\alpha_{13} f_{2}\left(s_{1}, s_{2}\right)$ contribution. 


\section{Conclusion}

We have characterized, as a Gaussian non-Markovian process, the trie size in a dynamic list structure. The limiting process is a superposition of a deterministic function (of order $n$ ) and a Gaussian process (of order $\sqrt{n}$ ). As a further work, we intend to analyze the asymmetric trie $(p \neq q)$. The m-any trie should also be studied (sce Pittel [16]). In cooperation with D. Gardy, we are also presently considering some other applications of the techniques introduced here, notably to join sizes in dynamic relational data bases, which is an important open problem.

\section{Acknowledgments}

The author is indebted to R. Schot for suggesting the problem we have investigated here. C. Kenyon made some useful comments on an earlier version of the paper. We are grateful to Ph. Flajolet and C. Lefèvre for several discussions on this topic.

\section{References}

[1] Csaki, E., Földes, A. and Salminen, P. On the Joint Distribution of the Maximum and its Location for a Linear Diffusion; Ann. Inst. Henri Poincaré, 1987, 23, 2, 179-194.

[2] Daniels, H.E. and Skyrme, T.H.R. The Maximum of a Random Walk whose Mean Path has a Maximum; Adv. Appl. Prob., 1985, 17, 85-99.

[3] Daniels, H.E. The Maximum of a Gaussian Process whose mean Path has a Maximum, with an Application to the Strength of Bundles of Fibres; Adv. Appl. Prob., 1989, 21, 315-333.

[4] Durbin, J. The First-Passage Density of a Continuous Ganssian Process to a General Boundary; J. Appl. Prob., 1985, 22, 99-122.

[5] Flajolet, Ph., Regnier, M., Sedgewick, R., Some uses of the Mellin integral transfrom in the analysis of algorithms, INRIA - Rapport de recherche 398, Mai 1985.

[6] Flajolet, Ph., Puech, C. and Vullemin, J. The Analysis of Simple List Structures, Inform. Sci., 1986, 38, 121-146.

[7] Flajolet, Ph., Françon, J and Vulllemin, J. Sequence of operations analysis for dynamic data structures, J. Algorithms, 1980, 1, 111-141.

[8] JaCqueT, P. and REgnier, M., Limiling distributions for trie parameters; INRIA, Report 502.

[9] Jacquet, P. and Regnibr, M., Trie partitioning process: limiting distributions; Proceedings CAAP'86, LNCS 214, 196-210.

[10] Johnson, N.L. and Kotz, S., Urn models and their application; Wiley, 1977. 
[11] LouchaRD, G., Random walks, Gaussian processes and list structures; TCS, 1987, 53, 99-124.

[12] Loucha RD, G., Brownian Motion and algorithms complexily; B.I.T., 1986, 26, $17-34$.

[13] Louchard, G., Schott, R. and Randrianarimanana, B. Dymamic Algorithms in D.E. Knuth's Model : a Probabilistic Analysis; Theor. Comp. Sc., $1992,93,201-225$.

[14] Lovcha RD, G., Schott, R. Probabilistic analysis of some distributed algorithms; Procedings CAAP'90, LNCS 431, 239-253 and Random Structures and Algorithuns, 1991, 2, 2, 151-185.

[15] Lovcha RD, G. Trie size in a dynamic list structure, Laboratoire d'Informatique Théorique, TR-252, 1992.

[16] Prttes, B., Patlis in a random digital tree: limiting distributions; Adv. Appl. Prob., 1986,18, 139-155.

[17] REgnier, M. and J Acquer, P., New results on the size of tries; IEEE-Tr. InI. Theor., 1989, 35, 1, 203-205. 